\title{
The HEP-COVID Trial
}

\author{
An Expert Interview with Alex C Spyropoulos ${ }^{1-3}$
}

1. The Donald and Barbara Zucker School of Medicine at Hofstra/Northwell, Hempstead, NY, USA; 2. The Institute for Health Innovations and Outcomes Research, the Feinstein Institutes for Medical Research, Manhasset, NY, USA; 3. Anticoagulation and Clinical Thrombosis Services, Northwell Health at Lenox Hill Hospital, New York, NY, USA

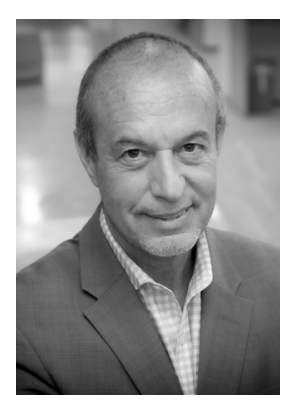

\begin{abstract}
Alex Spyropoulos
Alex C Spyropoulos, MD is a Professor of Medicine at Hofstra, Northwell School of Medicine, and System Director of Anticoagulation and Clinical Thrombosis Services for the multi-hospital Northwell Health System in New York. He is also a professor of the Center for Health Innovations and Outcomes Research, as part of the Feinstein Institute for Medical Research. He is co-chair of the Council on Leadership of Thrombosis at Northwell Health System. He is a Fellow of the American College of Physicians, American College of Chest Physicians, International Academy of Clinical and Applied Thrombosis/Haemostasis, and the Royal college of Physicians, Canada. Dr Spyropoulos has been involved as principal investigator, scientific committee member, steering and executive committee member, or member of the data safety monitoring board in multiple international, multicentre randomized trials in thrombosis and anticoagulant therapy, including the National Heart, Lung and Blood Institute (NHLBI) BRIDGE and NHLBI Kids DOTT studies and the Canadian Institutes of Health Research (CIHR) PAUSE trial. He was the co-principal investigator for MARINER, a global phase III multicentre study of thromboprophylaxis in medical patients, and he is the principal investigator of the HEP-COVID trial of thromboprophylaxis in hospitalized patients with coronavirus disease 2019 (COVID-19), and the CORE-19 registry of post-discharge outcomes in patients with COVID-19. He is a steering committee member for World Thrombosis Day as part of the International Society of Thrombosis and Haemostasis (ISTH), a steering committee member of the North American Thrombosis Forum, Chair of the Scientific and Standardization Committee of ISTH on the diagnosis, prevention and treatment of COVID-19 thrombosis, and co-author or member of the eighth, ninth and tenth 2021 American College of Clinical Pharmacy Guidelines on perioperative antithrombotic therapy and senior author for the 2013 International Consensus Statement on venous thromboembolic disease. He is Section Editor for Thrombosis and Haemostasis, and is on the editorial staff for Hospital Medicine. He has been published in over 250 peer-reviewed journals, including 20 publications on COVID-19-related topics in leading journals, such as The New England Journal of Medicine, Blood, Blood Advances, Circulation and The Lancet. He is a reviewer for many journals. He received his medical degree from the University of Pennsylvania School of Medicine in Philadelphia, PA. He completed his internship and residency in internal medicine at the University of New Mexico Health sciences Center in Albuquerque, NM. He is board certified in internal medicine.
\end{abstract}

\section{Keywords}

COVID-19, hospitalized, thromboembolism, heparin, low-molecular-weight heparin, mortality

Disclosures: Alex C Spyropoulos discloses research grants from Janssen and Boehringer Ingelheim and consulting fees from Janssen, Bayer, Boehringer Ingelheim, Portola, Bristol Myers Squibb, Sanofi and the ATLAS Group.

Acknowledgements: The author wishes to acknowledge the assistance of D Mark Goldin, who is co-first author of the study. The trial was dedicated to the memory of Dr Will R Hiatt, MD, who passed during the course of the trial and whose efforts as President of the Colorado Prevention Center were instrumental during trial initiation activities. Dr Spyropoulos also wishes to acknowledge Professor Jim Douketis, Chair of the Data Safety Monitoring Board, and its members, Professors Sam Schulman and John Kittelson, who tirelessly devoted their time to the trial. He would also like to thank the many patients and their families who participated in this trial during the very difficult time of the COVID-19 pandemic. The study results were presented as a Latest Science abstract at the European Society of Cardiology Congress, 27-30 August 2021. Medical writing assistance was provided by Katrina Mountfort, freelancer, and funded by Touch Independent Medical Education.

Review process: This is an expert interview and, as such, has not undergone the journal's standard peer review process.

Compliance with ethics: This article is an interview and did not involve any studies with human or animal subjects performed by any of the authors.

Data availability: Data sharing is not applicable to this article as no datasets were generated or analyzed during the current study/during the writing of this article.

Authorship: The named author meets the International Committee

of Medical Journal Editors (ICMJE) criteria for authorship of this

manuscript, takes responsibility for the integrity of the work as a whole,

and has given final approval for the version to be published.

Received: 7 September 2021

Published online: 21 October 2021

Citation: Heart International. 2021;15(2):62-4

Corresponding author: Alex Spyropoulos, Northwell Health at Lenox Hill Hospital, 130 E 77th St, New York, NY 10075 USA. E: aspyropoul@northwell.edu

Support: No funding was received for the publication of this article.
C oronavirus disease 2019 (COVID-19) has been associated with significant risk of thromboembolic events, particularly among hospitalized patients with elevated D-dimer levels. ${ }^{1-2}$ Recently, the COVID-19 Registry (CORE-19) study found that discharge anticoagulants were associated with a substantial decrease in the risk of thromboembolic disease or death in the post-discharge period. ${ }^{3}$ However, controversy has persisted around the optimal anticoagulation strategies. The HEP-COVID phase III trial (Full dose heparin vs. prophylactic or intermediate dose heparin in high risk COVID-19 patients; ClinicalTrials. gov identifier: NCT04401293) aimed to investigate the optimal dosage of low molecular weight heparin (LMWH) in hospitalized patients with COVID-19 and high thromboembolic risk. ${ }^{4}$

In an expert interview, Alex Spyropoulos, who designed and led this study, discusses the study aims, design and findings, which have led to a paradigm shift in the care of high-risk hospitalized patients with COVID-19.

\section{Q. What were the aims, design and patient population of the HEP-COVID trial?}

The HEP-COVID trial was designed to answer the following question: could high-risk hospitalized patients with COVID-19 (i.e. those at risk of major thromboembolic events and death) benefit from a therapeutic dose of thromboprophylaxis of anticoagulation or standard-of-care doses of heparin? It used a typical thromboprophylactic clinical trial design. Other trials have addressed the question of whether therapeutic anticoagulation is beneficial as an add-on treatment to reduce the disease severity of COVID-19 in hospitalized patients. HEP-COVID tried to answer a different question about whether therapeutic doses of anticoagulation, using an agent that is well established, have the potential to reduce thromboembolic outcomes and mortality, compared with standard-of-care heparin. Another important part of the clinical trial design was that, at the 
time of randomization, we stratified patients as requiring intensive care unit (ICU) or non-ICU levels of care.

The inclusion criteria of the trial are unique and very important. Participants were hospitalized patients with a diagnosis of COVID-19 by nasal swab or serologic testing. There was a requirement for supplemental oxygen as per investigator judgement. Very importantly, the D-dimer level was greater than four times the upper limit of normal, and around $99 \%$ of the trial participants entered the trial based on D-dimer criteria. By using D-dimer as an entry criterion, we were able to identify a very high-risk group of patients with COVID-19. In the control group, the baseline incidence of major thromboembolism and death was almost $42 \%$, which was reduced to $28.7 \%$. So not only were these participants very high risk, but disease progression of thromboembolism was modifiable using LMWH. This is very different to the multi-platform trials and all the other published data in ICU populations, such as the INSPIRATION trial (Intermediate-dose vs standard prophylactic anticoagulation and statin vs placebo in ICU patients with COVID-19; ClinicalTrials.gov identifier: NCT04486508) and the ACTION trial (Full Anticoagulation Versus Prophylaxis in COVID-19: COALIZAO ACTION Trial; ClinicalTrials.gov identifier: NCT04394377)..$^{5-8}$

\section{Q. What were the findings of the trial?}

The primary outcome was major thromboembolism, including venous thromboembolism, arterial thromboembolism and all-cause mortality, up to day 30 . We also looked at components of the primary outcome, which included major thromboembolism and all-cause mortality. The secondary outcomes were a composite of the primary outcome measures, but during the hospitalization phase of the trial, up to day 14

The primary outcome in the modified intention-to-treat (MITT) population, which was the population studied first, and who received at least one dose of study medication, showed a significant $32 \%$ risk reduction in the composite primary efficacy outcome with a therapeutic dose of LMWH, compared with institutional standard-of-care heparin, which translated to a $13 \%$ absolute risk reduction. The majority of treatment effects were seen in the non-ICU strata. In the patients stratified to non-ICU care, there was a significant risk reduction (per protocol) of $54 \%$ in the primary outcome measure for therapeutic dose LMWH, which represented around a $20 \%$ absolute risk reduction, with statistical significance. When looking at the components of the primary outcome measure, we saw a very significant, approximately 63\% relative risk reduction, a 19\% absolute risk reduction, of major thromboembolism with therapeutic dose LMWH $(p<0.0001)$. For all-cause mortality, although there were no significant differences between the two groups, there were numerically fewer deaths in the therapeutic dose group (25 versus $31 ; n=56$ ), and many of these were cardiovascular deaths.

In terms of secondary efficacy outcomes, we also saw a significant $36 \%$ relative risk reduction (13\% absolute risk reduction) in the primary efficacy outcome at day 14, i.e. in-hospital days rather than in the post-discharge phase of the trial. This did not come at a price of increased bleeding; there were no statistically significant differences between the groups in terms of major bleeding. In the entire study, there were eight major bleeding events; two in the standard dose group and six in the therapeutic dose group. In the non-ICU patients, there were two major bleeds in each group, i.e. no signal of major bleeds. In the ICU patients, there were no major bleeds in the standard dose group, but four in the therapeutic dose group.

In summary, this was a very important trial that definitively answered the hypothesis that therapeutic dose LMWH reduces thromboembolism and death in high-risk hospitalized patients with COVID-19. This was primarily seen in the non-ICU stratum, and almost all the treatment effect was driven by a reduction in thromboembolic events. As mentioned above, this trial population was unique in having such a high risk of thromboembolic events, which gives us an insight into the mechanism of thrombosis in these patients. This is a 'just-in-time' treatment paradigm, where we identified a high-risk group of patients that were about to enter cytokine storm, a hyperinflammatory state, but not as far advanced as the ICU populations, in which patients are so far advanced in their inflammatory and cytokine storm that increasing anticoagulation does nothing to alter their disease outcomes.

\section{Q. What is the clinical significance of these findings?}

These findings are exciting, as they have enabled us to identify, for the first time, a very high-risk subgroup of patients with COVID-19, whose disease is modifiable using therapeutic dose LMWH. This trial will provide a new standard of care for thromboprophylaxis in hospitalized patients with COVID-19. I expect that the relative risk reductions that we saw are translatable to less acutely ill hospitalized patients with COVID-19, but not the absolute risk reductions. In terms of absolute risk reductions in non-ICU patients, we have a number needed to treat (NNT) of 5. This is something I have never seen in my 25 years as a thrombologist; usually we would be happy with an NNT of 50-300. The number needed to harm in the non-ICU stratum was 2,000. These are incredible numbers, and demonstrate that the HEP-COVID trial has given us a new paradigm for primary prevention of thrombosis in hospitalized patients with COVID-19. This is the first clinical trial using a classical antithrombotic trial design to show that therapeutic doses of LMWH reduces the composite endpoint of major thrombosis and death without causing bleeding in high-risk patients with COVID-19.

\section{Q. What questions remain unanswered and what future studies are needed?}

There is a lot more work to be done in ICU patients. This trial, as well as the multi-platform trials, have demonstrated clearly that increasing the intensity of anticoagulation does nothing to improve outcomes in ICU patients, and also results in huge amounts of harm in terms of major bleeding. In terms of future studies, we need novel strategies to change the thrombotic response in patients, such as contact pathway activation inhibitors, complement activation inhibitors, combination therapy with antiplatelet and anticoagulants and fibrinolytic drugs. We need out-of-the-box, forward-thinking paradigms to look at how we can decrease the thrombotic risk in the ICU population.

\section{Q. What have been the challenges of conducting a clinical trial during a pandemic?}

This has been the most difficult trial I have conducted. To put it in perspective, we recently finished a global study of 12,000 patients, which was nowhere near as difficult as conducting this trial during a pandemic. There was a bias among frontline clinicians to start therapeutic doses of LMWH, although there was no clinical evidence for its use, so it was considered unethical to randomize patients during the trial. This was the first hurdle to overcome. Secondly, even something as simple as the consent process was a huge challenge, as this is typically done using a paper form, but we wanted to minimize investigator-patient contact. Thirdly was the logistics: there was a flow of investigators that were being constantly redeployed to other hospitals, as well as a constant flow of patients in and out of other units or to different hospitals during the course of the trial. This meant that investigators could change on a daily basis, or patients could be transferred to new facilities every two days. 
Lastly, overcoming the bias of the frontline clinicians caused controversy; I was on the front page of a New York Times Magazine article entitled "The Covid Drug Wars That Pitted Doctor vs. Doctor", where there was tension between clinical researchers and frontline clinicians. ${ }^{9}$ They were treating the patient in front of them, whereas I was treating the next thousand patients that were just like the patient of the frontline clinician, and we needed both to work. We also need to remember that this was an extremely high-risk patient group, which we can see by looking at the all-cause mortality: about 1 in 5 patients died during the course of the trial. $\square$
1. Klok FA, Kruip MJHA, van der Meer NJM, et al. Confirmation of the high cumulative incidence of thrombotic complications in critically ill ICU patients with COVID-19: an updated analysis. Thromb Res. 2020;191:148-50.

2. Spyropoulos AC, Weitz Jl. Hospitalized COVID-19 patients and venous thromboembolism: a perfect storm. Circulation. 2020;142:129-32.

3. Giannis D, Allen SL, Tsang J. Postdischarge thromboembolic outcomes and mortality of hospitalized patients with COVID-19: the CORE-19 registry. Blood. 2021:137:2838-47.

4. Goldin M, Giannis D, Diab W, et al. Treatment-dose LMWH

versus prophylactic/intermediate dose heparins in high-risk COVID-19 inpatients: Rationale and design of the HEP-COVID
Trial. Thromb Haemost. 2021. doi: 10.1055/a-1475-2351. Online ahead of print.

5. Sadeghipour P, Talasaz AH, Rashidi F, et al. Effect of intermediate-dose vs standard-dose prophylactic anticoagulation on thrombotic events, extracorporea membrane oxygenation treatment or mortality amon patients with COVID-19 admitted to the intensive care unit: The ClinicalTrials.gov. Intermediate-dose vs Standard Prophylac Anticoagulation and Statin vs Placebo in ICU Patients With COVID-19 (INSPIRATION). ClinicalTrials. gov Identifier: NCT04486508. Available at: https://clinicaltrials.gov/ct2/show/ NCT04486508 (accessed 14 September 2021.
7. Lopes RD, de Barros E Silva PGM, Furtado RHM, et al. Therapeutic versus prophylactic anticoagulation for patients admitted to hospital with COVID-19 and elevated D-dimer concentration (ACTION): An open-label, multicentre, randomised, controlled trial. Lancet. 2021;12;397:2253-63.

8. ClinicalTrials gov. Full Anticoagulation Versus Prophylaxis in COVID-19: COALZAO ACTION Trial (ACTION). ClinicalTrials gov COVID-19: COALIZAO ACTION Trial (ACTION). ClinicalTrials.gov dentifier: NCT04394377. Available at: https://clinicaltrials.go ct2/show/NCT04394377 (accessed 14 September 2021).

9. Dominus S. The Covid Drug Wars That Pitted Doctor vs. Doctor New York Times Magazine, 2020. Available at: www.nytimes. com/2020/08/05/magazine/covid-drug-wars-doctors.html (accessed 13 September 2021). 